\title{
SUBTIPOS DE ANSIEDAD SOCIAL EN POBLACIÓN ADULTA E INFANTO-JUVENIL: DISTINCIÓN CUANTITATIVA VERSUS CUALITATIVA
}

\author{
IHAB ZubeidAT, JuAn C. SiERRA y ANTONIO FERnÁNDEZ-PARRA \\ Facultad de Psicología, Universidad de Granada
}

\begin{abstract}
Resumen: El trastorno de ansiedad social ha sido menos investigado que los otros trastornos de ansiedad. Todavía se tiene escasa información sobre las diferencias y similitudes entre las dos formas, específica y generalizada, de este trastorno en la población adulta, y menos todavía en la infanto-juvenil. Por ello, en el presente estudio teórico se describen las principales investigaciones relacionadas con estas dos formas realizadas en población adulta y adolescente. También se analizan los intentos de algunos autores por ofrecer una distinción cuantitativa y/o cualitativa de estas dos formas de ansiedad social para una mejor diferenciación de las mismas. Las conclusiones indican una vaga separación entre los subtipos específico y generalizado de la ansiedad social, señalando la necesidad de llevar a cabo más estudios que pretenden recobrar el conocimiento asociado a la definición, etiología, psicopatología y tratamiento de la ansiedad social y de sus subtipos.
\end{abstract}

Palabras clave: Subtipos, ansiedad social, población adulta, niños y adolescentes, estudio teórico.

\section{Subtypes of social anxiety in adult and child-adolescent population: Quantitative versus qualitative distinction}

\begin{abstract}
The social anxiety disorder has been less investigated than other anxiety disorders. There is still very little information in adult population about the differences and similarities between the specific and generalized forms of this disorder, and still less in children and adolescents. For that reason, in the present review principals studies carried in adult and adolescent population about these two forms are described. Also, attempts of some authors to offer a quantitative and/or qualitative distinction of both social anxiety subtypes are analyzed for a better differentiation of them. The conclusions indicate a lazy separation between specific and generalized subtypes of social anxiety. This determine the necessity of more studies that pretend regain the knowledge associated to the definition, etiology, psychopathology and treatment of social anxiety and its subtypes.
\end{abstract}

Keywords: Subtypes, social anxiety, adult population, children and adolescents, theoretical study.

\section{INTRODUCCIÓN}

Uno de los aspectos más importantes del comportamiento humano son las relaciones interpersonales, siendo imprescindibles para la supervivencia al igual que otras necesidades como el agua, la comida y el sexo. El miedo social de las personas se puede localizar a lo largo de un continuo, en el que los niveles de ansiedad dan lugar a conductas que oscilan desde la inhibición en algunas situaciones

Recibido: 9 mayo 2006; aceptado 30 enero 2007.

Correspondencia: Ihab Zubeidat, Departamento de Personalidad, Evaluación y Tratamiento Psicológico, Facultad de Psicología, Universidad Nacional de Educación a Distancia, Juan del Rosal 10, 28040 Madrid. Correo-e: izubeidat@psi.uned.es específicas (hablar en público, enfrentar a figuras de autoridad, etc.) hasta la evitación de cualquier relación nueva. En esta línea, distintas investigaciones defienden la existencia de un continuo en el que se localiza la timidez en un extremo como el menor grado de ansiedad social, pasando por la ansiedad social específica y generalizada como niveles patológicos intermedio y alto, respectivamente, y llegando a la ansiedad social generalizada junto al trastorno de personalidad evitadora en otro extremo, correspondiendo con los niveles más elevados de patología y representando a la forma más grave del problema (Chavira, Stein y Malcarne, 2002).

Especificar el nombre exacto del problema de ansiedad social resulta polémico. En el pasa- 
do casi siempre se ha denominado como fobia social; no obstante, en la actualidad, se emplea el término de trastorno de ansiedad social. Por nuestra parte, optamos por la denominación de ansiedad social en lugar de fobia social a lo largo de esta revisión, reflejando niveles patológicos (altos, intermedios o bajos) de ansiedad social entre los sujetos de los distintos estudios que vamos a describir a continuación. Como consecuencia, resulta más adecuado considerar este problema como un complejo trastorno de ansiedad social, en vez de etiquetarlo como fobia social; esto permite al investigador una mejor comprensión de este trastorno, especialmente cuando lo comparamos con otros trastornos de ansiedad, teniendo éste una gran utilidad desde una perspectiva de especie y evolutiva, tal como defendían algunos autores como Botella, Baños y Perpiñá (2003). A su vez, sus formas etiquetadas como ansiedad social específica y generalizada harían referencia a dos dimensiones con niveles de ansiedad social diferentes, pudiendo comparar el grado de patología de cada una de ellas con la otra. En esta línea, el DSM-IV (American Psychiatric Association, 1994) incluye el nombre alternativo de trastorno de ansiedad social, manteniendo connotaciones que le caracterizan ser más generalizado e incapacitante que las provenientes de la categoría fobia social.

El trastorno por ansiedad social ha sido menos investigado que otros trastornos de ansiedad durante el siglo pasado. Con la publicación del DSM-III en 1980 (American Psychiatric Association, 1980), este trastorno fue incluido como entidad diagnóstica independiente y caracterizado por el miedo exagerado e irracional a la observación o al escrutinio de los demás a situaciones específicas asociadas al rendimiento, tales como hablar en público, usar el aseo o escribir en presencia de otras personas. Mientras tanto, el trastorno de personalidad por evitación mantenía características tales como la hipersensibilidad al posible rechazo, retraimiento social, baja autoestima y deseo de recibir afecto y aceptación. Por ello, los pacientes que sufrían el trastorno de personalidad por evitación, que evitaban distintas situaciones sociales, se excluyeron del diagnóstico de ansiedad social. Por su parte, el DSM-III-R
(American Psychiatric Association, 1987) introdujo un subtipo generalizado de ansiedad social como respuesta al hecho de que muchos individuos presentaban miedo a interactuar en distintas situaciones sociales (Liebowitz, Gorman, Fyer y Klein, 1985), concretamente en la mayoría de las mismas.

Este cambio, junto a la eliminación de la exclusión arbitraria del DSM-III de los cuadros clínicos que coincidían con los criterios del trastorno de personalidad por evitación, dio lugar a que se ampliara el campo de la ansiedad social. El temor al desconcierto o a la humillación en el subtipo generalizado es prácticamente a cualquier situación social (hablar con desconocidos, compañeros, etc.), diferenciándose de su subtipo complementario que careció de denominación, lo que dio lugar a que se llamara de diferentes nombres, tales como «no generalizado», «específico» «de actuación» o «aislado» (Heimberg, Holt, Schneier, Spitzer y Liebowitz, 1993). La ansiedad social generalizada fue conceptualizada en el DSMIII-R como un miedo constante a la mayoría de las situaciones sociales, mejorando de esta manera los criterios diagnósticos de la ansiedad social (véase Sandín, 1997). Estos cambios producidos en el DSM-III-R no han ayudado a identificar los límites entre ambos trastornos. Además, la confusión creada en torno a esta situación sigue sin tener una solución en el DSM-IV y DSM-IV-TR (American Psychiatric Association, 2000) a pesar de que los criterios asociados al trastorno de personalidad por evitación han pasado a centrarse en el malestar en las relaciones más que en la interacción social por sí.

Actualmente, se admite la existencia de dos tipos de ansiedad social: ansiedad social simple (discreta) y ansiedad social generalizada (Lépine y Chignon, 1994). Por su parte, la Organización Mundial de la Salud (OMS) incluye, por primera vez, la ansiedad social como categoría diagnóstica independiente a su sistema de clasificación de los trastornos mentales y del comportamiento, el CIE-10, en 1992 (Organización Mundial de la Salud, 1992); se pone énfasis en el miedo que experimentan las personas por ser el foco de atención o el temor a manifestar una serie de comportamientos humillantes o emba- 
razosos. Tanto el DSM-IV como el DSM-IVTR conciben la ansiedad social como el «miedo persistente y acusado a situaciones sociales o actuaciones en público por temor a que resulten embarazosas». Estas dos últimas ediciones especifican un subtipo generalizado de la ansiedad social definido como un miedo que abarca la mayoría de situaciones sociales, considerando el diagnóstico adicional del trastorno de personalidad por evitación, el cual fue excluido en las ediciones anteriores (DSM-III, DSM-III-R y CIE-10). Hoy por hoy, la ansiedad social generalizada y el trastorno de personalidad por evitación representan variantes cuantitativas psicopatológicas, sin llegar a ser trastornos cualitativamente diferenciados, ya que éste último guarda una multitud de similitudes con la ansiedad social.

De todo ello, destacamos que las características de las dos formas específica y generalizada de ansiedad social, especialmente en lo que respecta a prevalencia, factores sociodemográficos, características psicopatológicas y comorbilidad han sido menos investigadas en la población infanto-juvenil que en la adulta. Concretamente, en España los trabajos que se han preocupado por abordar este objetivo son escasos. Dada la cuestionable existencia de subtipos de ansiedad social y la escasez de estudios realizados sobre la distinción de los mismos en las poblaciones adulta e infantojuvenil, la investigación debería responder a las preguntas planteadas con respecto a las diferencias y similitudes entre los diferentes subtipos de la ansiedad social propuestos en estas dos poblaciones. Por ello, el objetivo principal de este estudio teórico (Montero y León, 2005) es ofrecer una descripción detallada de los principales estudios relativos a las dos formas, específica y generalizada, de ansiedad social (explicando los distintos criterios de separación entre las mismas, los cuales dependen de cada investigador o grupo de investigadores), tanto en población adulta como infanto-juvenil, así como reflejar la problemática creada en torno a la distinción cuantitativa versus cualitativa entre ambas formas. Por último, se ofrecerá una serie de conclusiones relacionadas con la situación actual de la distinción de ambas formas.

\section{SUBTIPOS DE LA ANSIEDAD SOCIAL}

\author{
Ansiedad social generalizada versus \\ específica en adultos
}

Los trabajos actuales sobre la ansiedad social se preocupan por el abordaje de diferentes subtipos de la misma, ya que desde el DSM-III-R se considera la existencia de un subtipo generalizado caracterizado por el miedo a la mayoría de las situaciones sociales (American Psychiatric Association, 1987). Paradójicamente, no se sabía el número de situaciones sociales necesarias para establecer un diagnóstico de ansiedad social del subtipo generalizado. Por defecto, aparece otro subtipo denominado por diferentes autores (Levin et al., 1993; Liebowitz et al., 1988; Turner, Beidel y Townsley, 1992) como «específico», «no generalizado», «circunscrito» o «limitado». Esta separación inicial permitió que en la mayoría de los estudios realizados al respecto en población adulta se identifiquen grupos de ansiedad social con niveles distintos de patología asociada, dependiendo del criterio utilizado por cada autor o grupo autores. La Tabla 1 presenta un resumen de distintos estudios que han utilizado diferentes criterios de separación desde una orientación cuantitativa versus cualitativa, de acuerdo a los instrumentos de evaluación empleados y a las características de las muestras escogidas (normal versus clínica o/y española versus norteamericana), obteniendo distintas denominaciones o categorías de ansiedad social.

Salvo en los dos estudios de Stein y Deutsch (2003) y Turner et al. (1992), todos los autores que aparecen en la Tabla 1 proponen un criterio de separación correspondiente a la orientación cuantitativa para clasificar a sus sujetos con ansiedad social, basándose en el número de situaciones sociales temidas por los mismos. Los autores que adoptaron la orientación cualitativa clasificaron a los individuos con ansiedad social basándose en el contenido (por ejemplo, situaciones de interacción social versus actuación social) de las situaciones sociales productoras de miedo. Es decir, todos estos estudios empíricos han operacionalizado las propuestas teóricas sobre los subtipos de ansiedad social de acuerdo a distintos criterios crea- 
Tabla 1. Denominación de los subtipos de ansiedad social en población adulta

\begin{tabular}{|c|c|c|}
\hline Autores & Denominación o categoría & Orientación \\
\hline $\begin{array}{l}\text { American Psychiatric Association } \\
(1987,1994 \text { y 2000) }\end{array}$ & $\begin{array}{l}\text { Subtipo generalizado } \\
\text { Subtipo no generalizado }\end{array}$ & Cuantitativa \\
\hline Chavira y Stein (2002) & $\begin{array}{l}\text { Subtipo generalizado } \\
\text { Subtipo no generalizado }\end{array}$ & Cuantitativa \\
\hline Furmark et al. (2000) & $\begin{array}{l}\text { Subtipo generalizado } \\
\text { Subtipo no generalizado } \\
\text { Subtipo discreto }\end{array}$ & Cuantitativa \\
\hline Heimberg y Holt (1989) & $\begin{array}{l}\text { Subtipo generalizado } \\
\text { Subtipo circunscrito } \\
\text { Subtipo no generalizado }\end{array}$ & Cuantitativa \\
\hline Kessler et al. (1994) & $\begin{array}{l}\text { Miedo a hablar en público } \\
\text { Resto de los miedos sociales }\end{array}$ & Cuantitativa \\
\hline Levin et al. (1993) & $\begin{array}{l}\text { Subtipo generalizado } \\
\text { Subtipo específico o no generalizado }\end{array}$ & Cuantitativa \\
\hline Liebowitz et al. (1988) & $\begin{array}{l}\text { Subtipo generalizado } \\
\text { Subtipo específico o no generalizado }\end{array}$ & Cuantitativa \\
\hline Mannuzza et al. (1995) & $\begin{array}{l}\text { Ansiedad social generalizada } \\
\text { Ansiedad social no generalizada }\end{array}$ & Cuantitativa \\
\hline Quero et al. (2003) & $\begin{array}{l}\text { Miedo a hablar en público } \\
\text { Ansiedad social circunscrita } \\
\text { Ansiedad social generalizada }\end{array}$ & Cuantitativa \\
\hline Stein (1997) & $\begin{array}{l}\text { Miedo a hablar en público } \\
\text { Miedo a hablar en público y otro más } \\
\text { Miedo a gran variedad de situaciones }\end{array}$ & Cuantitativa \\
\hline Stein y Chavira (1998) & $\begin{array}{l}\text { Subtipo generalizado } \\
\text { Subtipo no generalizado }\end{array}$ & Cuantitativa \\
\hline Stein y Deutsch (2003) & $\begin{array}{l}\text { Miedo a hablar en público } \\
\text { Miedo a la interacción }\end{array}$ & Cuantitativa \\
\hline Turner et al. (1992) & $\begin{array}{l}\text { Ansiedad social específica } \\
\text { Ansiedad social generalizada }\end{array}$ & Cuantitativa \\
\hline
\end{tabular}

dos en función de los objetivos de cada autor o grupo de autores. De esta manera, hubo diferencias notables entre los distintos estudios empíricos en la elección de los criterios de separación, los cuales dependían de distintas operacionalizaciones de la conceptualización de los subtipos de ansiedad social. Este hecho nos permite obtener una amplia gama de resultados que aumentan el conocimiento relativo a las distintas formas de ansiedad social, aunque, en muchas ocasiones, existen varias limitaciones a la hora de explicarlos e interpretarlos, lo que pone en duda su generalización a otros contextos. A esto se añade, en ocasiones, la confusión creada en torno a los propios criterios de separación establecidos debido a la vaga descripción y justificación de los mismos.

Turner et al. (1992) establecen una categoría de ansiedad social generalizada, incluyendo a los pacientes que temen fiestas o reuniones 
sociales, iniciar o mantener conversaciones, y otra de ansiedad social específica que recoge a los sujetos que manifiestan miedo sólo ante situaciones circunscritas, tales hablar en reuniones, comer o escribir en público. Es decir, las personas que presentan miedo con más frecuencia a situaciones generales que requieren una interacción social prolongada con los demás obtendrían el diagnóstico de ansiedad social generalizada, mientras que las que muestran este temor únicamente ante situaciones de actuación social concretas y breves recibirían el diagnóstico de ansiedad social específica. En muestras clínicas, generalmente, se acepta la existencia de dos subtipos de ansiedad social, denominados como no generalizado y generalizado (Chavira y Stein, 2002; Stein y Chavira, 1998). Así, Mannuzza et al. (1995) establecen una distinción entre dos subtipos de trastorno por ansiedad social: generalizado y no generalizado, argumentando que el primer subtipo tiene la suficiente fiabilidad y validez para considerarlo como una categoría independiente y distinta de la forma no generalizada, pudiendo aludir a una variante familiar del trastorno de ansiedad social. Por su parte, Heimberg y Holt (1989) utilizan un criterio basado en la operacionalizan de la expresión «miedo a la mayoría de situaciones» que define la ansiedad social generalizada según el DSM-IV; estos autores obtienen tres subtipos de ansiedad social: uno primero llamado subtipo generalizado donde se incluyen aquellos individuos que presentan miedo a la mayoría o todas las situaciones sociales, uno segundo denominado subtipo discreto o circunscrito que recoge a los sujetos que muestran miedo ante una o dos situaciones sociales concretas, y; por último, uno tercero llamado subtipo no generalizado como una categoría intermedia donde el problema se sitúa entre el subtipo discreto y el generalizado, haciendo referencia al miedo a un número limitado de situaciones de interacción social.

Por otra parte, Kessler et al. (1994) clasifican a los miedos sociales en dos categorías: una primera caracterizada sólo por el temor a hablar en público, y otra segunda, que recoge al resto de los miedos sociales. Se ha demostrado que las dos categorías («miedo a hablar en público» $\mathrm{y}$ «miedo a la interacción») recogen a ítems parecidos entre sí en cuanto a contenido dentro de cada una de ellas, pero difieren entre sí cuando se comparan estas dos categorías (Stein y Deutsch, 2003); este hallazgo sugirió que el miedo a hablar en público y el miedo a la interacción representan dominios distintos de miedo a situaciones sociales. Estos dos subtipos mantienen semejanzas relativas a la edad de inicio, historia familiar y algunas características sociodemográficas, aunque el miedo a la interacción es más crónico, más perjudicial y mantiene mayor comorbilidad con otros trastornos que el miedo a hablar en público. Kessler et al. (1994) defienden que, realmente, no está muy clara la diferenciación entre los dos grupos descritos anteriormente, aunque afirman que el miedo a hablar en público tiene mucha más prevalencia que los demás miedos sociales, ya que una mayor parte de los pacientes con ansiedad social muestran únicamente el mismo.

Recientemente, ha habido otros intentos por delimitar subtipos de ansiedad social. Por ejemplo, Furmark, Tillfors, Stattin, Ekselius y Fredrikson (2000) realizan un análisis y clasificación de las personas de una muestra comunitaria basándose en cuatro variables: malestar, número de situaciones sociales, grado de deterioro funcional y número de criterios cumplidos para el trastorno de personalidad por evitación. De acuerdo con la puntuación obtenida en el conjunto de las variables, los individuos fueron clasificados en tres categorías: subtipo generalizado (puntuación alta), no generalizado (puntuación media) y ansiedad social discreta (puntuación baja). Por su parte, Stein et al. (2001) hacen uso de doce situaciones sociales (de las cuales la mitad son de actuación y la otra son de relación) con el objetivo de contabilizar la frecuencia de su ocurrencia en una muestra comunitaria; estos autores proponen un criterio cuantitativo basado en el DSM-IV, donde los sujetos fueron clasificados en un subtipo de ansiedad social generalizado cuando experimentaban miedo en siete o más situaciones sociales y en otro no generalizado que recoge al resto de los individuos. No obstante, a raíz del trabajo anterior se concluyó que no se puede hablar de categorías claramente diferenciadas en la población general pese a que dicha distinción entre los subtipos puede ser utilizada en la práctica clínica. 
Hay tan sólo un trabajo, realizado por Quero, Baños, Botella y Gallardo (2003) en población española, que tiene como objetivo delimitar subtipos de ansiedad social, basado en una muestra clínica de una edad comprendida entre 18 y 63 años; estos autores definen a grupos de ansiedad social mediante el análisis de conglomerados de las situaciones de la Anxiety Disorders Interview Schedule-Revised (ADIS-R), donde los individuos que mostraban miedo a un número elevado de situaciones sociales manifestaban un nivel mayor de psicopatología. Como resultado, se obtuvo tres subtipos de ansiedad social: uno primero que incluye a los individuos que temen únicamente situaciones de la ADIS-R referentes al miedo a hablar en público, otro segundo llamado ansiedad social circunscrita que engloba a las personas que temen un número limitado de situaciones sociales de la ADIS-R no referentes a hablar en público, y; uno tercero denominado ansiedad social generalizada que recoge a los sujetos que muestran temor a la mayoría de las situaciones de la ADIS-R.

Los estudios realizados en adultos se han centrado en las diferencias que se han observado entre pacientes con ansiedad social generalizada y otras fobias (discreta, especifica, no generalizada, etc.), basándose en características sociodemográficas (Heimberg, Hope, Dodge y Becker, 1990), tipo y severidad de los síntomas (Gelernter, Stein, Tancer y Uhde, 1992; Holt, Heimberg, Hope y Liebowitz, 1992), nivel de funcionamiento (Heimberg et al., 1990), inicio de la ansiedad social (Holt et al., 1992) y en los autoinformes, la observación y las respuestas fisiológicas a cambios comportamentales (Levin et al., 1993). Estos dos subtipos mantienen semejanzas relativas a la edad de inicio, historia familiar y algunas características sociodemográficas, aunque los miedos distintos al de hablar en público son más crónicos y perjudiciales, y mantienen mayor comorbilidad con otros trastornos que el miedo a hablar en público. Se ha demostrado que la ansiedad social generalizada tiene un comienzo más temprano, acompañada de bajos niveles de escolarización, lo que causa mayores niveles de ansiedad y depresión en actividades de realización social (Heimberg et al., 1990; Turner et al., 1992).
Además, los individuos con ansiedad social generalizada sufren mayores niveles de severidad clínica general y de interferencia que los de la no generalizada (Holt et al., 1992; Turner et al., 1992).

Todo lo anterior indica que la ansiedad social puede tomar dos formas: específica y generalizada. La primera se refiere a una serie de estímulos concretos productores de ansiedad, tales como hablar, comer, beber o escribir en público, mientras que la segunda está asociada a una variedad de situaciones sociales, en las que la ansiedad social generalizada se manifiesta en la mayor parte de las interacciones interpersonales (Mattick y Peters, 1988). Las personas que padecen ansiedad social del tipo generalizado tienen marcada su vida social por el miedo a que los demás les observen sudar, enrojecer, temblar o cometer actos ridículos, respondiendo con conductas evitativas inadecuadas, tales como evitar la mirada, hablar entre dientes o susurrar. Hay que señalar que la mayoría de los estudios anteriores han sido realizados en población adulta, mientras que la investigación en población infanto-juvenil es todavía muy escasa, tal como reflejaremos a continuación.

\section{Ansiedad social generalizada versus especifica en niños y adolescentes}

La importancia de las relaciones sociales en el desarrollo evolutivo y en el funcionamiento psicológico, social y académico ha despertado el interés por el estudio de las dificultades de adaptación social de los niños y adolescentes (Trianes, Jiménez y Muñoz, 1997). Sin embargo, el reconocimiento de dichas dificultades en esta población como un trastorno etiquetado ansiedad social ha sido un hecho reciente (Beidel, Ferell, Alfano y Yeganeh, 2001). De hecho, la American Psychiatric Association (2000) ha conceptualizado el trastorno de ansiedad social, incluyendo ciertos criterios diagnósticos relativos a la población infanto-juvenil. Los niños y adolescentes con ansiedad social suelen tener distintos problemas de ansiedad en el entorno escolar, tales como hablar en público, comer en la cafetería o cambiarse de ropa en la clase de 
deporte, etc. Algunos estudios (Essau, Conradt y Petermann, 1999; Wittchen, Stein y Kessler, 1999) informan de una prevalencia de la ansiedad social infanto-juvenil que oscila entre el 1,6 y el $10 \%$.

Según afirma Sandín (1997), la distinción entre la ansiedad social específica y generalizada, especificada en el DSM-IV, apenas ha sido estudiada en población infanto-juvenil; no obstante, los resultados obtenidos señalan que, entre los niños y adolescentes, predomina el miedo a múltiples situaciones, especialmente las relacionadas con hablar en público. Por otro lado, Sandín, Charot, Valiente y Santed (1998) informaron que las niñas presentan niveles significativamente más altos que los niños en la frecuencia y la intensidad del miedo; además, ambos informan tener niveles elevados en la dimensión de temores a peligros físicos y muerte. También, se encontraron correlaciones moderadas entre el miedo y las variables de sensibilidad a la ansiedad, rasgo se ansiedad y afectividad negativa en población infanto-juvenil (Valiente, Sandín y Chorot, 2002), resultando las correlaciones presentadas entre el miedo y la sensibilidad a la ansiedad superiores que las halladas entre el mismo y otras variables. Otros resultados revelan que las niñas muestran más miedos y preocupaciones de tipo social que los niños (Anderson, Williams, McGee y Silva, 1987). Así, las chicas presentan más casos de timidez (Stevenson-Hinde y Glover, 1996) y ansiedad social que los chicos, en muestras comunitarias, aunque no en clínicas.

La extensión del deterioro en niños y adolescentes que padecen ansiedad social es muy amplia. Los que presentan ansiedad social circunscrita, rara vez se encuentran incapacitados, siendo limitado su sufrimiento, mientras que los del tipo generalizado evitan una gran variedad de situaciones sociales, repercutiendo negativamente en su funcionamiento cotidiano (Ballesteros y Conde, 1999). Una de las situaciones que evitan los individuos con ansiedad social y que supone un problema potencialmente significativo es la escuela. Se ha demostrado que casi la mitad de los sujetos de una muestra clínica con fobia escolar reciben el diagnóstico de ansiedad social (Francis, Strauss y Last, 1987). Como resultado, estos menores pierden importantes oportunidades normativas y evolutivas durante su desarrollo académico y socioemocional. Además, se ha demostrado que el trastorno de ansiedad social en la población infanto-juvenil suele estar asociado con pocos amigos, soledad, estado de ánimo deprimido (Beidel, Turner y Morris, 1999), rechazo a la escuela (Last, Hersen, Kazdin, Orvaschel y Perrin, 1991), deficiente rendimiento en la escuela (Beidel, 1991) y consumo de alcohol (DeWitt, MacDonald y Offord, 1999).

Se han realizado pocos estudios en población infanto-juvenil con el objetivo de establecer un criterio que sirva para diferenciar entre los dos subtipos (generalizado y no generalizado) de la ansiedad social. Hofmann et al. (1999) establecieron un criterio necesario para el diagnóstico del subtipo generalizado que requiere informar de una moderada ansiedad (una puntuación de 4 , donde 8 es la puntuación máxima) en todas las situaciones sociales más temidas por los sujetos; de lo contrario, tendría lugar el diagnóstico de ansiedad social no generalizada. Por su parte, Wittchen et al. (1999) realizan un estudio en una muestra comunitaria de adolescentes y jóvenes adultos, donde la asignación de los individuos al subtipo generalizado de la ansiedad social requería reunir los criterios diagnósticos del DSM-IV, manifestar miedo a tres o más de las situaciones evaluadas por la M-CIDI (Munich-Composite Internacional Diagnostic Interview) y reconocer que el miedo y la evitación de dichas situaciones estaban presentes desde su inicio por parte del sujeto. Estos autores encuentran que los chicos y chicas adolescentes con ansiedad social del subtipo generalizado suelen tener una edad de inicio más temprana, presentar mayor deterioro funcional y persistencia de las respuestas alteradas, así como aportar tasas más altas de tratamiento psicológico o médico.

Recientemente, Zubeidat, Salinas, Sierra y Fernández-Parra (2007) han clasificado a una muestra de 1.012 adolescentes escolarizados, de acuerdo a un criterio cuantitativo basado en el análisis de las puntuaciones criterio obtenidas en la Social Interaction Anxiety Scale (SIAS), obteniendo tres grupos pertinentes a tres cluster claramente diferenciados (adolescentes $\sin$ ansiedad social $(n=568)$, otros con 
miedos sociales leves $(n=340)$ y un tercer grupo con ansiedad social $(n=104))$. Dentro del tercer cluster de se han determinado dos subtipos de ansiedad social (específico y generalizado), donde se aplicó un criterio cuantitativo a los 104 jóvenes que puntuaron por encima del punto corte establecido en la SIAS $(40,34)$. El subtipo específico $(n=66)$ reunió a los jóvenes que obtuvieron un punto de corte igual o mayor de 40.34 y que además contestaron a diez o menos situaciones sociales (ítems de la SIAS) con alta ansiedad (valorada en 3 ó 4). Por su parte, el subtipo generalizado $(n=38)$ incluyó a los sujetos que además de tener un punto corte igual o mayor de 40.34 respondieron con alta ansiedad a once o más situaciones sociales de la SIAS.

Los niños y adolescentes que sufren ansiedad social generalizada infravaloran su capacidad cognoscitiva y experimentan malestar que repercute negativamente en su funcionamiento cotidiano, refiriendo una multitud de situaciones ansiosas (Beidel, 1991). Determinados problemas como el déficit en las habilidades sociales e interacción social también son característicos de la ansiedad social generalizada. En esta línea, se ha encontrado que los adolescentes con ansiedad social generalizada, miedo a hablar en público, introvertidos e inestables emocionales, manifiestan más dificultades interpersonales que otros que no padecen dichos trastornos y los extravertidos (Inglés, Méndez e Hidalgo, 2001). Además, la ansiedad social generalizada presenta mayor comorbilidad con otros trastornos psicopatológicos que la específica, siendo éstos más graves y crónicos en el caso de la primera y teniendo un inicio más temprano que en el caso de la segunda (Pine, Cohen, Gurley y Ma, 1998). La ansiedad social generalizada se presenta siempre de la misma forma a lo largo de la infancia y la adolescencia hasta la vida adulta, al contrario de lo que ocurre en la específica, donde se manifiesta una discontinuidad a través del tiempo (Conde y Ballesteros, 1995). Recientemente, Olivares, Piqueras y Rosa (2006) informan, en una muestra de adolescentes españoles, de diferencias estadísticamente significativas entre los dos subtipos (específico y generalizado) de la ansiedad social respecto al género y a medidas de ansiedad, evitación social, miedo a la evaluación negativa y sus correlatos, donde el subtipo generalizado superó al específico.

Por su parte, la ansiedad social específica se ha diferenciado de la ansiedad generalizada en determinados aspectos destacados por Strauss y Last (1993); los niños que padecen ansiedad social del subtipo específico, a diferencia de los aquejados de fobias específicas, se caracterizan por ser más miedosos, sufrir más aislamiento social y sentimientos de depresión, y tener más edad. En la Tabla 2 se muestra un resumen a modo de un listado de las situaciones sociales productoras de miedo, basándose en su frecuencia de ocurrencia. Por un lado, Beidel y Randall (1994) ordenaron las situaciones sociales ansió-

Tabla 2. Frecuencia de las situaciones sociales ansiógenas en población infanto-juvenil

\begin{tabular}{clc}
\hline \multicolumn{1}{c}{ Autores } & \multicolumn{1}{c}{ Situación } & Porcentaje \\
\hline & Hablar formalmente en público & $89 \%$ \\
& Comer en presencia de otros & $39 \%$ \\
Beidel y Randall (1994) & Participar en fiestas & $28 \%$ \\
& Conversar con personas con autoridad & $21 \%$ \\
& Hablar con un estilo informal & $13 \%$ \\
\hline \multirow{3}{*}{ Strauss y Last (1993) } & Participar en situaciones escolares & $64 \%$ \\
& Hablar en público & $57 \%$ \\
& Temer situaciones productoras de rubor & $25 \%$ \\
& Comer o beber ante los demás & $18 \%$ \\
& Vestirse en presencia de otras personas & $14 \%$ \\
& Usar aseos públicos & $7 \%$ \\
\hline
\end{tabular}


genas de su estudio según su frecuencia de ocurrencia, resultando la situación «Hablar formalmente en público» la más frecuente $(89 \%)$ y «Hablar con estilo informal» la menos frecuente $(13 \%)$. Por otro, Strauss y Last (1993) informaron que «Participar en situaciones escolares» ocurría con alta frecuencia (64\%), mientras que «Usar aseos públicos» mostraba baja frecuencia (7\%) (Véase Sandín, 1997). Respecto a la frecuencia de los síntomas somáticos, Beidel, Neal y Lederer (1991) muestran que la taquicardia, los temblores, el rubor y la sudoración junto a las náuseas presentaron una frecuencia de ocurrencia de $70 \%, 67 \%, 62 \%$ y $54 \%$ respectivamente.

En cuanto al contexto donde aparece la ansiedad social específica, se considera que el medio escolar es el más frecuente, en donde tienen lugar las relaciones de interacción con los compañeros que requieren comunicación verbal. Esto repercute de forma negativa en las tareas y funciones escolares de los niños y adolescentes, tales como el rendimiento escolar, la asistencia a clase y la realización de las actividades extraescolares. Por ello, la relación con los iguales y profesores se ve mermada, tanto si se trata de la ansiedad social específica como la generalizada, debido a las dificultades de interacción con ellos, aumentando éstas cuando se comprometen las habilidades sociales (Ballesteros y Conde, 1999).

\section{PROBLEMÁTICA DE LOS SUBTIPOS DE LA ANSIEDAD SOCIAL: LÍMITES Y CONCLUSIONES}

\section{Diferenciación cuantitativa versus cualitativa entre los dos subtipos y sus límites}

A algunas personas les generan miedo las situaciones sociales relativas a la actuación personal, mientras que a otras les inquietan más las situaciones sociales que tienen que ver con el trato social. Esta distinción entre ambos subtipos se ha recogido en algunas medidas de ansiedad social (Liebowitz, 1987). No obstante, Heimberg et al. (1999) defienden que esta diferenciación entre las situaciones sociales de actuación y de interacción es confusa, especialmente cuando se le pide a los sujetos que valoren el grado de temor y evitación que experimentan. También ha habido otros intentos de diferenciar los dos subtipos de la ansiedad social basados en la manera en que la ansiedad se generaliza a otras situaciones sociales temidas, ya que algunas personas tienen miedo a una única actividad (por ejemplo, hablar en público), mientras que otras manifiestan miedo a una multitud de situaciones interpersonales. Esta especificación referida al número de situaciones que generan temor en sujetos con ansiedad social se refleja en el DSM-III-R, DSM-IV y DSM-IV-TR (American Psychiatric Association, 1987, 1994, 2000) sobre un subtipo generalizado de ansiedad social distinto de otro tipo no generalizado.

La tarea de delimitar un subtipo de ansiedad social generalizado, tanto en población adulta como adolescente, en población clínica o comunitaria, pretende minimizar la variabilidad encontrada en el mismo. Se piensa que las personas a las que les afectan más las situaciones de actuación personal es posible que se quejen de un número más limitado de temores denominados no generalizados (Heimberg et al., 1990; Turner et al., 1992). No obstante, el problema que acarrea esta división es la falta de cuantificación exacta de «la mayoría de las situaciones» (Heimberg et al., 1993). Esta confusión aparece no sólo en la población adulta, sino también en la adolescente, donde se dispone de pocos datos al respecto; es decir, cada investigador operacionaliza la expresión «la mayoría de las situaciones sociales» según la estimación que consideran más adecuada. Las propuestas de clasificación (en su mayoría cuantitativas) que se han expuesto en la Tabla 1 constituyen un ejemplo claro del intento de varios autores por determinar subtipos de ansiedad social.

Un ejemplo de una propuesta de clasificación realizada mediante la combinación de distintos criterios es la de Olivares, Rosa y GarcíaLópez (2004), quienes agrupan a las personas por el número y la naturaleza de las situaciones temidas. El número de las situaciones sociales permite distinguir entre cuatro categorías 
distintas: ansiedad social específica, leve, moderada y gravemente generalizada. A su vez, la naturaleza de dichas situaciones da lugar a dos tipos de las mismas: las primeras no requieren la relación física con los otros, mientras que las segundas la conllevan como condición necesaria. Dentro de la categoría de «pacientes con ansiedad social» se da una gran variabilidad, dependiendo de la manera en la que éstos resultan afectados por el problema. Por ejemplo, el miedo a hablar en público es una variante de la ansiedad social que corresponde a su nivel más bajo, donde el problema para la mayoría de los sujetos que lo sufren aparece en momentos concretos de su vida, incluyéndose bajo la categoría «ansiedad social específica». Al contrario, en otros casos se produce un deterioro social que se va incrementando gradualmente conforme aumenta el número y el tipo de las situaciones sociales temidas, pasando por los niveles intermedios de ansiedad social que corresponden a las categorías «leve» y «moderada», y llegando al nivel elevado, donde tiene lugar la «ansiedad social generalizada».

Las críticas que se han realizado al subtipo del DSM-III-R fueron cuatro: vaga conceptualización de la frase «la mayoría de las situaciones sociales», validez cuestionable de una diferencia categórica basada en la cantidad de las situaciones temidas en lugar de su calidad, ausencia de pruebas concluyentes capaces de realizar una diferenciación cualitativa entre subtipos y de un subtipo intermedio que identifique a sujetos con temor a distintas situaciones interpersonales, pero no a la mayoría de las mismas. La solución de estos problemas era el objetivo del Grupo de Trabajo del DSM-IV, abordando la fiabilidad y validez de otros sistemas de subclasificación. En esta línea, Schneier et al. (1998) encontraron que la ansiedad resultante de la actuación sin miedo significativo a ninguna situación interpersonal se manifestaba en el 6\% de una muestra de 229 pacientes, el miedo a una o dos situaciones se presentaba en el $24 \%$ y el miedo a tres o más situaciones se daba en el $70 \%$ de los casos. No obstante, no se han observado diferencias significativas relativas a los porcentajes del subtipo generalizado, concebido unas veces como temor a «la mayoría de las situaciones» y otras como miedo a «tres o más situaciones de interacción social».

Algunos autores (Holt et al., 1992; Turner et al., 1992) pretendieron investigar las diferencias entre los dos subtipos de ansiedad social, verificando que los sujetos del subtipo generalizado puntúan más alto en un amplio campo de la misma y otras medidas de autoinforme que las personas aquejadas del específico. Además, los primeros experimentaron alta interferencia en la vida y un mayor nivel de severidad clínica general que los segundos. Parece ser que la posible diferenciación del subtipo generalizado de ansiedad social concretado en el DSM-IIIR, DSM-IV y DSM-IV-TR de otros subtipos de la misma es una cuestión de grado, es decir, las diferencias son cuantitativas. No obstante, el estudio de Heimberg et al. (1990) señala posibles diferencias de tipo cualitativo entre las personas del subtipo no generalizado que sufrían miedo a hablar en público, manifestando una reactividad cardiaca notablemente más baja que los del subtipo generalizado ante una actividad que supone un reto comportamental individualizado. Al contrario, Turner et al. (1992) demostraron que no existen diferencias entre los sujetos con ansiedad social generalizada y específica en cuanto al ritmo cardiaco manifestado ante la prueba de conducta estandarizada, aunque realmente los pacientes del subtipo generalizado presentan un ritmo cardiaco ligeramente más alto que los del específico ante la misma.

Las preguntas actuales se dirigen hacia una distinción más sólida de los dos subtipos de ansiedad social. Concretamente, ¿están estos subtipos mejor conceptualizados como variantes del mismo trastorno o constituyen distintas taxonomías con características fenotípicas similares? (Hofmann, 2000). Se piensa que el subtipo generalizado constituye una forma más incapacitante de la ansiedad social (American Psychiatric Association, 1994, 2000), ya que se caracteriza por tener un patrón disperso de miedos sociales, mientras que la forma específica es mucho menos incapacitante al ser más limitada, pudiendo presentarse el temor a una única situación social (Schneier, Johnson, Horing, Liebowitz y Weissman, 1992). Esta segunda forma no está reconocida como un subtipo en 
el DSM-IV y el DSM-IV-TR, pero la literatura se ha referido a ella (Heimberg et al., 1993), denominada a veces como «ansiedad social circunscrita» (McNeil, 2001).

Por su parte, tanto el DSM-IV como el DSM-IV-TR no han ofrecido unas directrices firmes que faciliten el diagnóstico de la ansiedad social generalizada, es decir, no han limitado el número exacto de las situaciones sociales que dan lugar a la misma. Además, si nos guiamos únicamente por el criterio cuantitativo, la gran mayoría de los individuos obtendría el diagnóstico de ansiedad social no generalizada, ya que es más frecuente que tengan un sólo miedo o dos que cinco o más (Baños, Botella, Guillén y Benedito, 2003). Algunos autores (Hofmann, 2000; McNeil, 2001) interpretan que la distinción entre los dos subtipos es cuantitativa y que no está diferenciada por categorías, sino que existe un continuo de severidad. La forma generalizada junto al trastorno de personalidad por evitación representan el polo más grave, la generalizada sin dicho trastorno es una forma menos grave que la anterior, la específica por si sola representa la forma menos severa y los individuos no ansiosos se sitúan en el polo opuesto del continuo. Hofmann (2000) usa este continuo para proponer que la forma generalizada junto al trastorno de personalidad por evitación constituyen la forma más complicada del trastorno de ansiedad social, mientras que la específica es la más simple. Esta manera de entender la relación entre ambas formas de ansiedad social alude a la distinción cuantitativa.

En contraste con la diferenciación cuantitativa, la cualitativa propone que dichos dos subtipos comparten algunas similitudes fenotipícas y no llegan a constituir trastornos diferentes (McNeil, 2001). Los defensores de la propuesta cualitativa indican que el número de situaciones sociales temidas no es la base de la diferenciación de los subtipos de ansiedad social. Hook y Valentiner (2002) definen los dos subtipos en términos de la difusión de los miedos sociales, estando la ansiedad social específica asociada a un número de miedos más limitado que la generalizada; la ansiedad social generalizada es entendida como el miedo a situaciones que requieren la interacción con los otros en situaciones de realización social, mientras que la específica es concebida como el miedo a una situación determinada de rendimiento que requiere una actuación concreta ante los demás. Además, la distinción cualitativa señala que estos dos subtipos mantienen diferencias relativas a sus causas y al pronóstico (Hook y Valentiner, 2002). Parece ser que el tipo generalizado está más estrechamente asociado con las situaciones de interacción, mientras que el específico lo hace con las situaciones de rendimiento (Stemberger, Turner, Beidel y Calhoun, 1995). A su vez, los miedos relacionados con la interacción y el rendimiento se distinguen y mantienen correlatos consistentes con los de la ansiedad social generalizada y específica, respectivamente (Hooke, Valentiner y Kim, 2000).

Como conclusión, los datos relativos a la distinción cuantitativa versus cualitativa entre la ansiedad social específica y generalizada son contradictorios, careciendo de claridad la diferenciación entre ambas. No obstante, la ansiedad social generalizada se asocia con niveles más altos de deterioro funcional que la específica.

\section{Discusión y conclusiones}

La existencia de subtipos de ansiedad social ha sido cuestionada por falta de un criterio de separación consistente debido a la falta de claridad en la conceptualización de la ansiedad social generalizada en los distintos manuales de clasificación, así como a la confusión de la misma con el trastorno de personalidad por evitación. Con el cambio del DSM-III al DSM-III$\mathrm{R}$ se describió la ansiedad social generalizada como el miedo a interactuar en una o más situaciones sociales, excluyendo los criterios clínicos relativos al trastorno de personalidad por evitación, concebido éste como el malestar y el miedo a la evaluación negativa experimentados en las situaciones de interacción social y no tanto en los contactos personales. Este hecho permitió alcanzar una mejor distinción entre las dos formas de ansiedad social, ampliando el campo de estudio de la misma, aunque siguió sin estar bien definida la ansiedad social generalizada, ya que el miedo era a cualquier situa- 
ción social. Además, estos cambios dificultaron la delimitación de los límites entre la ansiedad social generalizada y el trastorno de personalidad por evitación, tal como se reflejó en la introducción. Esta problemática no se ha solventado en el DSM-IV y el DSM-IV-TR a pesar de que los cuadros clínicos de este trastorno estaban asociados al malestar en las relaciones personales que en la interacción social en sí. Es decir, tanto la ansiedad social generalizada como el trastorno de personalidad por evitación constituyen variantes psicopatológicas cuantitativas no diferenciadas cualitativamente, guardando estos dos trastornos más similitudes que diferencias entre ellos. La inclusión de la ansiedad social generalizada en una categoría independiente fue más tarde en el CIE-10. Con la llegada del DSM-IV y DSMIV-TR, la ansiedad social generalizada fue descrita como el miedo a la mayoría de las situaciones sociales, considerando el diagnóstico adicional del trastorno de ansiedad por evitación. En la actualidad, se reconoce la existencia de dos subtipos de ansiedad social, aunque éstos están recibiendo poca atención por la comunidad científica.

Los estudios realizados sobre la distinción entre la ansiedad social generalizada y la ansiedad social específica son escasos en la población adulta; la mayoría de ellos (American Psychiatric Association, 1987, 1994, 2000; Chavira y Stein, 2002; Furmark et al., 2000; Heimberg y Holt, 1989; Kessler et al., 1994; Levin et al., 1993; Liebowitz et al., 1988; Mannuzza et al., 1995; Stein, 1997; Stein y Chavira, 1998; Stein y Deutsch, 2003; Turner et al., 1992) tuvieron lugar en muestras norteamericanas y tan solo uno (Quero et al., 2003) se basó en una muestra española. El criterio de separación propuesto por una gran parte de estos autores ha sido cuantitativo, donde los dos subtipos, específico y generalizado, de la ansiedad social han sido diferenciados según el número de situaciones sociales temidas, autoinformadas por los sujetos de las diferentes muestras utilizadas. Estos intentos de separación cuantitativa entre dichos subtipos se deben claramente a la vaga definición del subtipo generalizado de la ansiedad social en la American Psychiatric Asociation, conceptualizado como el «miedo a la mayoría de las situaciones sociales», estando confusa su distinción del subtipo específico. Este hecho hizo que cada autor propusiera su propio criterio de separación, dependiendo de los distintos instrumentos de evaluación empleados y de las características de las muestras de sujetos seleccionadas. Por su parte, la literatura científica casi carece de trabajos que han adoptado una orientación de tipo cualitativa. En esta línea, se menciona el estudio epidemiológico de Wittchen (1998), quien informa que la forma generalizada tiene menor prevalencia que la específica y presenta mayor comorbilidad y un mayor grado de deterioro. Esto indica la necesidad de realización de otros estudios epidemiológicos sobre la ansiedad social con el objetivo de avanzar más en el conocimiento asociado a la diferenciación entre los dos tipos de ansiedad social en cuanto a ciertas variables, tales como la tasa de prevalencia, factores sociodemográficos, psicopatología y comorbilidad.

Evidentemente, si el estudio de la ansiedad social como un trastorno de ansiedad ha sido relativamente descuidado en adultos (Liebowitz et al., 1985), es casi completa la falta de literatura en menores. Aunque, los trastornos de ansiedad de los niños y adolescentes han recibido atención clínica e investigadora en los últimos años, todavía es escasa la información relativa a la ansiedad social de los niños y adolescentes. Concretamente, en la población infanto-juvenil existen aún menos estudios relativos a la diferenciación de las dos formas (específica y generalizada) de la ansiedad social; además, la mayoría de ellos están realizados en muestras anglosajonas, salvo algunos en poblaciones de habla española (Olivares, 2004; García-López, 2000; Ramos, 2004; Ruiz, 2003). Estas carencias señalan que las futuras investigaciones deberían responder a las preguntas planteadas con respecto a las diferencias y similitudes entre los diferentes subtipos de la ansiedad social propuestos (especialmente, entre el generalizado y específico), con más énfasis, en la población de niños y adolescentes.

Existe una limitación en los estudios que pretenden diferenciar los dos subtipos, consistente en el uso de definiciones distintas para los 
mismos, lo que dificulta la comparación entre diferentes trabajos; además, una gran parte de los datos obtenidos en los estudios epidemiológicos son contradictorios. Actualmente, tal como indica el International Consensus Group todavía no se sabe si existen únicamente diferencias de tipo cuantitativo (referentes a gravedad) o también cualitativo entre los dos subtipos de la ansiedad social. Tampoco se sabe si la forma no generalizada es propensa a volverse más grave y acercarse a la generalizada, y si la intervención temprana es capaz de prevenir el avance de la misma (Ballenger et al., 1998). Hoy por hoy, la distinción más común entre ambos subtipos es cuantitativa, ya que los datos empíricos concluyentes relativos a la distinción cualitativa son escasos, tales como los del estudio de Turner et al. (1992), donde la clasificación entre los dos subtipos de ansiedad social se hace en base al contenido de los ítems o de las situaciones de actuación e interacción social. A pesar de la posible diferenciación cualitativa futura entre las dos categorías de ansiedad social, se señala que las pruebas de evaluación disponibles en estos momentos llevan a realizar una interpretación prudente de tipo cuantitativo y no cualitativo entre ellas (Heimberg et al., 1990; Turner et al., 1992).

Uno de los aspectos del que tienen que preocuparse los futuros esfuerzos de investigación en la ansiedad social es la operacionalización de la misma (incluyendo a sus subtipos) y de otros conceptos que se solapan con ella, tales como la timidez o la personalidad evitadora. Diferentes estudios han pretendido hacer una aproximación al significado de la timidez y la ansiedad social para determinar las dificultades de relación social referentes a cada uno de ellas (Greco y Morris, 2001; Monjas y Caballo, 2002). Asimismo, Olivares et al. (2002) proponen que las investigaciones futuras centradas específicamente en la población infanto-juvenil deberían hacer uso de estudios longitudinales con el objetivo de observar el curso de la operacionalización rigurosa, tanto de la timidez como de la ansiedad social. En esta línea, la timidez se ha concebido como el miedo a la evaluación negativa en situaciones de interacción social y la evitación o el retraimiento ante situaciones que incluyen a gente conocida, poco conocida o desconocida (Buss, 1980), relacionándose este concepto con otros términos como el retraimiento social (Rubin y Stewart, 1996). Por último, sin duda la detección temprana del trastorno de ansiedad social en la etapa infanto-juvenil, encauzaría al profesional a alcanzar una cierta eficacia mediante la exposición y el entrenamiento en habilidades sociales.

En resumen, la incertidumbre creada en torno a la definición, prevalencia, etiología, psicopatología y el tratamiento de la ansiedad social y de sus dos formas (específica y generalizada) marcará las próximas líneas de investigación en el terreno de la misma.

\section{REFERENCIAS BIBLIOGRÁFICAS}

American Psychiatric Association (1980). Diagnostic and statistical manual of mental disorders (3rd ed.). Washington, DC: Autor.

American Psychiatric Association (1987). Diagnostic and statistical manual of mental disorders (3rd ed., rev.). Washington, DC: Autor.

American Psychiatric Association (1994). Diagnostic and statistical manual of mental disorders (4th ed.). Washington, DC: Autor.

American Psychiatric Association (2000). Diagnostic and statistical manual for mental disorders (cuarta edicióntexto revisado) (DSM-IV-TR). Washington, DC: Autor.

Anderson, J. C., Williams, S., McGee, R., y Silva, P. A. (1987). DSM-III disorders in preadolescent children. Archives of General Psychiatry, 44, 69-76.

Ballenger, J. C., Davidson, J. R. T., Lecrubier, Y., Nutt, D. J., Bobes, J., Beidel, D. C., Ono, Y., y Westenberg, H. G. (1998). Consensus statement on panic disorder from the International Consensus Group on Depression and Anxiety. Journal of Clinical Psychiatry, 59, 54-60.

Ballesteros, M. C., y Conde, V. J. M. (1999). Trastorno por ansiedad social de inicio en la infancia y la adolescencia. En J. G, Bobes, M. P., González, P. A., Sáiz y G. Bousoño (Eds.), Abordaje actual del trastorno por ansiedad social (pp. 35-62). Barcelona: Masson.

Baños, R. M., Botella, C., Guillén, V., y Benedito, M. (2003). Fobia social: comorbilidad y diagnóstico diferencial. En C. Botella, R. M. Baños y C. Perpiñá (Eds.), Fobia social. Avances en la psicopatología, la evaluación y el tratamiento psicológico del trastorno de ansiedad social (pp. 37-64). Barcelona: Paidós.

Beidel, D. C. (1991). Social phobia and overanxious disorder in school-age children. Journal of the American Academy of Child and Adolescent Psychiatry, 4, 545552. 
Beidel, D. C., Ferrell, C., Alfano, C. A., y Yeganeh, R. (2001). The treatment of childhood social anxiety. The Psychiatric Clinics of North America, 24, 831-846.

Beidel, D. C., Neal, A. M., y Lederer, A. S. (1991). The feasibility and validity of a daily diary for the assessment of anxiety in children. Behavior Therapy, 22, 505-517.

Beidel, D. C., Turner, S. M., y Morris, T. L. (1999). Psychopathology o childhood social phobia. Journal of the American Academy of Child and Adolescent Psychiatry, 38, 643-650.

Berg, I., y Jackson, A. (1985). Teenage school refusers grow up: A follow-up study of 168 subjects, ten years on average after inpatient treatment. British Journal of Psychiatry, 147, 366-370.

Bernstein, G. A., Garfinkel, B. D., y Hoberman, H. M. (1989). Self-reported anxiety in adolescents. American Journal of Psychiatry, 146, 384-386.

Botella, C., Baños, R. M., y Perpiñá, C. (2003). Fobia social. Avances en la psicopatología, la evaluación y el tratamiento psicológico del trastorno de ansiedad social. Barcelona: Paidós.

Buss, A. H. (1980). Self-consciousness and social anxiety. San Francisco: Freeman.

Chambless, D. L., Baker, M. J., Baucom, D. H., Beutler, L. E., Calhoun, K. S., Christoph, P., Daiuto, A., DeRubeis, R., Detweiler, J., Haaga, D. A. F., Johnson, S. B., McCurry, S., Mueser, K. T., Hope, K. S., Sanderson, W. C., Shoham, V., Stickle, T., Williams, D. A., y Woody, S. L. (1998). Update on empirically validated therapies II. The Clinical Psychologist, 51, 3-16.

Chavira, D. A., y Stein, M. B. (2002). Combined psychoeducation and treatment with selective serotonin reuptake inhibotors for youth with generalized social anxiety disorder. Journal of Child and Adolescence Psychopharmacology, 12, 47-54.

Chavira, D. A., Stein, M. B., y Malcarne, V. L. (2002). Scrutinzing the relationship between shyness and social phobia. Journal of Anxiety Disorders, 16, 585589.

Conde, V. J. M., y Ballesteros, M. C. (1995). La psicopatología y la psiquiatría infanto-juveniles desde la perspectiva del adulto. En J. Rodríguez Sacristán (Ed.), Psicopatología del niño y del adolescente (pp. 1017-1068). Tomo II. Secretariado de Publicaciones de la Universidad de la Universidad de Sevilla.

DeWitt, D. J., MacDonald, K., y Offord, D. R. (1999). Childhood strees and symptoms on drug dependence in adolescence and early adulthood: Social phobia as a mediator. American Journal of Orthopsychiatry, 69, 61-72.

Essau, C. A., Conradt, J., y Petermann, R. (1999). Frecuency and comorbidity of social phobia and social fears in adolescents. Behavior Research and Therapy, $37,831-843$
Francis, G., Strauss, C. C., y Last, C. G. (1987). Social anxiety in school phobic adolescents. Documento presentado en el Annual Meeting of the Association for the Advancement of Behavior Therapy: Boston.

Furmark, T., Tillfors, M., Stattin, H., Ekselius, L., y Fredrikson, M. (2000). Social phobia subtypes in the general population revealed by cluster analysis. $P s y$ chological Medicine, 30, 1335-1344.

García-López, L. J. (2000). Un estudio de la eficacia entre tres modalidades de tratamiento para población adolescente con fobia social. Tesis doctoral no publicada. Facultad de Psicología. Universidad de Murcia.

Gelernter, C. S., Stein, M. B., Tancer, M. E., y Uhde, T. W. (1992). An examination of syndromal validity and diagnostic subtypes in social phobia and panic disorder. Journal of Clinical Psychiatry, 53, 23-27.

Greco, L. A., y Morris, T. L. (2001). Treating childhood shyness and related behavior. Empirically Evaluated approaches to promote positive social interactions. Clinical Child and Family Psychology Review, 4, 299-318.

Hazen, A. L., y Stein, M. B. (1995). Differential diagnosis and comorbidity of social phobia. En M. B. Stein (Ed.), Social Phobia: Clinical and research perspectives (pp. 3-41). Washington, DC: American Psychiatric Press Inc.

Heimberg, R. G., Holt, C. S., Schneier, F. R., Spitzer, R. L., y Liebowitz, M. R. (1993). The issue of subtypes in the diagnosis of social phobia. Journal of Anxiety Disorders, 7, 249-269.

Heimberg, R. G., y Holt, C. S. (1989). The issue of subtypes in the diagnosis of social phobia: A report to the social phobia subgroup for DSM-IV. Manuscrito no publicado.

Heimberg, R. G., Hope, D. A., Dodge, C. A., y Becker, R. E. (1990). DSM-III-R subtypes of social phobia: Comparison of generalized social phobics and public speaking phobics. Journal of Nervous and Mental Disease, 178, 172-179.

Heimberg, R. G., Horner, K. J, Juster, H. R., Safren, S. A., Brown, E. J., Schneier, F. R. \& Liebowitz, M. R. (1999). Psychometric properties of the Liebowitz Social Anxiety Scale. Psychological Medicine, 29, 199-212.

Hofmann, S., G. (2000). Treatment of social phobia: Potential mediators and moderators. Clinical Psychology: Science and Practice, 7, 3-16.

Hofmann, S. G., Albano, A. M., Heimberg, R. G., Tracey, S., Chorpita, B. F., y Barlow, D. H. (1999). Subtypes of social phobia in adolescents. Depression and Anxiety, 9, 15-18.

Holt, C. S., Heimberg, R. G., Hope, D. A., y Liebowitz, M. R. (1992). Situational domains of social phobia. Journal of Anxiety Disorders, 6, 63-77.

Hooke, J. N., y Valentiner, D. P. (2002). Are Specific and Generalized Social Phobias Qualitatively Distinct? Clinical Psychology: Science and Practice, 9, 379-395. 
Hooke, J. N., Valentiner, D. P., y Kim, H. (2000). Distinction of social phobia subtypes. Poster aceptado en el 34 del Annual Convention of the Association of the Advancement of Behavior Therapy. New Orleans: LA. Inglés, C., Méndez, F. X., e Hidalgo, M. D. (2001). Dificultades interpersonales en la adolescencia: ¿Factor de riesgo de fobia social? Revista de Psicopatología y Psicología Clínica, 6, 91-104.

Kashani, J. H., Orvaschel, H., Rosenberg, T. K., y Reid, J. C. (1989). Psychopathology in a community sample of children and adolescents: A developmental perspective. Journal of the American Academy of Child and Adolescent Psychiatry, 28, 701-706.

Kessler, R., McGonagle, K. A., Zhao, S. Nelson, C. B., Hughes, M., Eshleman, S., Wittchen, H. U., y Kendler, K. S. (1994). Lifetime and 12-month prevalence of DSM-III psychiatric disorders in the United States: Results from the National Comorbidity Survey. Archives of General Psychiatry, 51, 8-19.

Last, C. G., Hersen, M., Kazdin, A., Ovaschel, H., y Perrin, S. (1991). Anxiety disorders in children and their families. Archives of General Psychiatry, 48, 928-934.

Lépine, J. P., y Chignon, J. M. (1994). Sémiologie des troubles anxieux et phobiques. Encyclopédie Médico-Chirurgicale. Tome I. 37-112-A-10.

Levin, A. P., Saoud, J. B. Strauman, T. Gorman, J. M., Fyer, A. J., Crawford, R., y Liebowitz, M. R. (1993). Responses of «generalized» and «discrete» social phobics during public speaking. Journal of Anxiety Disorders, 7, 207-222.

Liebowitz, M. R. (1987). Social phobia. Modern Problems in Pharmacopsychiatry, 22, 141-173.

Liebowitz, M. R., Gorman, J., Fyer, A. J., Campeas, R., Levin, A., Sandberg, D., Hollander, E., Papp, L., y Goetz, D. (1988). Pharmacotherapy of social phobia: An interim report of a placebo-controlled comparison of phenelzine and atenolol. Journal of Clinical Psychiatry, 49, 252-257.

Liebowitz, M. R., Gorman, J., Fyer, A. J., y Klein, D. F. (1985). Social phobia: Review of a neglected anxiety disorder. Archives of General Psychiatry, 42, 729-736.

Mannuzza, S., Schneier, F. R., Champan, T. F., Liebowitz, M. R., Klein, D. F., y Fyer, A. J. (1995). Generalized social phobia: Reliability and validity. Archives of General Psychiatry, 52, 230-237.

Mattick, R. P., y Peters, L. (1988). Treatment of severe social phobia: Effects of guided exposure with and without cognitive restructuring. Journal of Consulting and Clinical Psychology, 56, 251-260.

McNeil, D. W. (2001). Terminology and evolution of construct in social anxiety and social phobia. En S. G. Hofmann y P. M. DiBartolo (Eds.), From social anxiety to social phobia: Multiple perspectives (pp. 8-19). Needham Heights, MA: Allyn \& Bacon.
Monjas, I., y Caballo, V. E. (2002). Psicopatología y tratamiento de la timidez en la infancia. En V. C., M. A. Simón (Eds.), Manual de psicología clínica infantil y del adolescente. Trastornos específicos (pp. 271-297). Madrid: Pirámide.

Montero, I., y León, G. O. (2005). Sistema de clasificación del método en los informes de investigación en Psicología. International Journal of Clinical and Health Psychology, 5, 115-127.

Olivares, J. (2004). Introducción. En J. Olivares (Ed.), Programa IAFS. Protocolo para el tratamiento de la fobia social en la adolescencia (pp. 9-13). Madrid: Pirámide.

Olivares, J., García-López, L. J., Hidalgo, M. D., La Greca, A. M., Turner, S. M., y Beidel, D. C. (2002). A pilot study on normative data for tow social anxiety measures: The Social Phobia and Anxiety Inventory and the Social Anxiety Scale for Adolescents. Revista Internacional de Psicología Clínica y de la Salud / International Journal of Clinical and Health Psychology, 2, 467-476.

Olivares, J., Piqueras, J. A., y Rosa, A. I. (2006). Características sociodemográficas y psicológicas de la fobia social en adolescentes. Psicothema, 18, 207-212.

Olivares, J., Rosa, A. I., y García-López, L. J. (2004). Fobia social en la adolescencia. El miedo a relacionarse y a actuar ante los demás. Ediciones Pirámide. Madrid.

Organización Mundial de la Salud (OMS) (CIE-10) (1992). Décima revisión de la clasificación internacional de las enfermedades. Trastornos mentales y del comportamiento. Madrid: Meditor.

Pine, D. S., Cohen, P., Gurley, D., y Ma, Y. (1998). The risk for early-adulthood anxiety and depressive disorders in adolescents with anxiety and depressive disorders. Archives of General Psychiatry, 55, 56-64.

Quay, H. C., y La Greca, A. M. (1986). Disorders of anxiety, withdrawal, and dysphoria. En H. C. Quay y J. S. Werry (Eds.), Psychopathological disorders of childhood (3rd ed., pp. 73-110). Nueva York: John Wiley \& Sons.

Quero, S., Baños, R. M., Botella, C., y Gallardo, M. (2003). Delimitación de subtipos clínicos en la fobia social. Psicología Conductual, 11, 429-458.

Ramos, V. (2004). Efecto de la retroalimentación audiovisual en la eficacia del IAFS. Tesis doctoral no publicada. Facultad de Psicología, Universidad de Murcia. Murcia.

Rubin, K. H., y Stewart, S. L. (1996). Social withdrawal in childhood: Conceptual and empirical perspectives. Advences in Clinical Child Psychology, 17, 157-196.

Ruiz, J. (2003). Efecto del tamaño del grupo en la eficacia del IAFS. Tesis doctoral no publicada. Facultad de Psicología, Universidad de Murcia: Murcia.

Sandín, B. (1997). Fobia social. En B. Sandín (Ed.), Ansiedad, miedos y fobias en niños y adolescentes (pp. 157193). Madrid: Dykinson. 
Sandín, B., Charot, P., Valiente, R. M., y Santed, M. Á (1998). Frecuencia e intensidad de los miedos en los niños: datos normativos. Revista de Psicopatología y Psicología Clínica, 3, 15-25.

Schneier, F. R., Johnson, J., Hornig, C. D., Liebowitz, M. R., y Weissman, M. M. (1992). Social phobia: comorbidity and morbidity in an epidemiological sample. Archives of General Psychiatry, 49, 282-288.

Schneier, F. R., Liebowitz, M. R., Beidel, D., Garfinkel, R., Heimberg, R., Horning, C. D., Johnson, J., Juster, H., Law, K., Mannuza, S., Mattia, J. I., Oberlander, Orsillo, S., E., Turner, S. M., y Weissman, M. M. (1998). MacArthur data reanalysis for DSM-IV: Social phobia. En T. A. Wideger, A. J. Frances, H. A. Pincus, R. Ross, M. B. First, W. Davis y M. Klaine (Eds.), DSM-IV Source book (Vol. 4, pp. 307-328). Washington, DC: American Psychiatric Press.

Stein, M. B. (1997). Phenomenology and epidemiology of social phobia. International Clinical Psychopharmacology, 12, S23-S26.

Stein, M. B., y Chavira, D. A. (1998). Subtypes of social phobia and comorbidity with depression and other anxiety disorders. Journal of Affective Disorders, 50, S11-S16.

Stein, M. B., y Deutsch, R. (2003). In search of social phobia subtypes: Similarity of feared social situations. Depression and Anxiety, 17, 94-97.

Stein, M. B., Fuetch, M., Müller, N., Hötler, M., Lieb, R., y Wittchen, H. U. (2001). Social anxiety disorder and the risk of depression. A prospetive community study of adolescents and young adults. Archives of General Psychiatry, 58, 251-256.

Stemberger, R., Turner, S. M., Beidel, D. C., y Calhoun, K. S. (1995). Social phobia: An analysis of possible developmental factors. Journal of Abnormal Psychology, 104, 526-531.
Stevenson-Hinde, J., y Glover, A. (1996). Shy girl's simple phobia in children. Journal of Psychological Psychiatry, 37, 181-187.

Strauss, C. C., y Last, C. G. (1993). Social and simple phobias in children. Journal of Anxiety Disorders, 7, 141152.

Trianes, M. V., Jiménez, M., y Muñoz, A. (1997). Competencia social: su educación y tratamiento. Madrid: Pirámide.

Turner, S. M., Beidel, D. C., Borden, J. W., Stanley, M. A., y Jacob, R. G. (1991). Social Phobia: Axis I and II Correlates. Journal of Abnormal Psychology, 100, 102106.

Turner, S. M., Beidel, D. C., y Townsley, R. M. (1992). Social phobia: A comparison of specific and generalized subtypes and avoidant personality disorder. Journal of Abnormal Psychology, 101, 326-331.

Valiente, R. M., Sandín, B., y Chorot, P. (2002). Miedos comunes en niños y adolescentes: relación con la sensibilidad a la ansiedad, la afectividad negativa y la depresión. Revista de Psicopatología y de Psicología Clínica, 7,61-70.

Wittchen, H. U. (1998). Prevalence, risk factors and course of SAD. En Social Phobia: Symposium $9^{\text {th }}$ Congress of the AEP. Copenhague, 20-24 September.

Wittchen, H. U., Stein, M. B., y Kessler, R. C. (1999). Social fears and social phobia in a community sample of adolescents and young adults: prevalence, risk factors and comorbidity. Psychological Medicine, 29, 309323.

Zubeidat, I., Salinas, J. M., Sierra, J. C., y Fernández-Parra, A. (2007). Psychometric properties of the Social Interaction Anxiety Scale and separation criterion between Spanish youths with and without subtypes of social anxiety. Journal of Anxiety Disorders, 21, 603-624. 